\title{
Brisk walking compared with an individualised medical fitness programme for patients with type 2 diabetes: a randomised controlled trial
}

\author{
S. F. E. Praet • E. S. J. van Rooij • A. Wijtvliet • \\ L. J. M. Boonman-de Winter • Th. Enneking • \\ H. Kuipers • C. D. A. Stehouwer • L. J. C. van Loon
}

Received: 28 August 2007 / Accepted: 14 January 2008 /Published online: 23 February 2008

(C) The Author(s) 2008

\begin{abstract}
Aims/hypothesis Structured exercise is considered a cornerstone in type 2 diabetes treatment. However, adherence to combined resistance and endurance type exercise or medical
\end{abstract}

Electronic supplementary material The online version of this article (doi:10.1007/s00125-008-0950-y) contains supplementary material, which is available to authorised users.

S. F. E. Praet • A. Wijtvliet · H. Kuipers · L. J. C. van Loon Department of Human Movement Sciences,

Nutrition and Toxicology

Research Institute Maastricht (NUTRIM),

Maastricht University, Maastricht, the Netherlands

E. S. J. van Rooij

Department of Clinical Health Psychology,

Tilburg University, Tilburg, the Netherlands

\section{J. M. Boonman-de Winter}

Department of Epidemiology and Contract Research,

Stichting Huisartsen Laboratorium, Etten-Leur,

the Netherlands

Th. Enneking

Primary Healthcare Centre 'De Keen',

Etten-Leur, the Netherlands

\section{D. A. Stehouwer}

Department of Internal Medicine,

Academic Hospital Maastricht,

Maastricht, the Netherlands

\section{S. F. E. Praet $(\square)$}

Department of Human Movement Sciences,

Maastricht University, Universiteitssingel 50,

6229 ER Maastricht, the Netherlands

e-mail: Stephan.Praet@BW.unimaas.nl fitness intervention programmes is generally poor. Groupbased brisk walking may represent an attractive alternative, but its long-term efficacy as compared with an individualised approach such as medical fitness intervention programmes is unknown. We compared the clinical benefits of a 12-month exercise intervention programme consisting of either brisk walking or a medical fitness programme in type 2 diabetes patients.

Methods We randomised 92 type 2 diabetes patients (60土 9 years old) to either three times a week of 60 min brisk walking $(n=49)$ or medical fitness programme $(n=43)$. Primary outcome was the difference in changes in $\mathrm{HbA}_{1 \mathrm{c}}$ values at 12 months. Secondary outcomes were differences in changes in blood pressure, plasma lipid concentrations, insulin sensitivity, body composition, physical fitness, programme adherence rate and health-related quality of life.

Results After 12 months, 18 brisk walking and 19 medical fitness participants were still actively participating. In both programmes, 50 and $25 \%$ of the dropout was attributed to overuse injuries and lack of motivation, respectively. Intention-to-treat analyses showed no important differences between brisk walking and medical fitness programme in primary or secondary outcome variables.

Conclusions/interpretation The prescription of group-based brisk walking represents an equally effective intervention to modulate glycaemic control and cardiovascular risk profile in type 2 diabetes patients when compared with more individualised medical fitness programmes. Future exercise intervention programmes should anticipate the high attrition rate due to overuse injuries and motivation problems.

Keywords Blood pressure - Exercise · Hyperglycaemia . Lipid metabolism $\cdot$ Physical fitness $\cdot$ Randomised controlled clinical trial · Type 2 diabetes mellitus 


\section{Abbreviations}

\section{bpm beats per min}

HOMA homeostasis model assessment

ITT intention-to-treat

$\dot{V} \mathrm{O}_{2 \text { peak }}$ peak oxygen uptake capacity

$W_{\max } \quad$ maximum workload capacity

\section{Introduction}

Regular exercise has been identified along with diet and medication as one of the three components of good diabetes therapy [1]. Structured exercise intervention programmes have been reported to be as effective as pharmaceutical strategies in improving glycaemic control [2-5] and/or cardiovascular risk profile $[6,7]$ in type 2 diabetes patients. Despite the growing body of scientific evidence on the health benefits of exercise intervention, most meta-analyses report a lack of studies that have tried to assess the long-term efficacy of exercise intervention in type 2 diabetes patients [3-5, 7-9]. In general, exercise intervention studies implement endurance and resistance type exercise, supervised by a physical therapist [10-17]. The long-term adherence to these (so-called) medical fitness programmes has been shown to range between 10 and $80 \%$ [11, 18-20]. Financial costs per patient are considerable in such programmes. Brisk walking exercise has been proposed as a less expensive alternative, with a good clinical outcome when patients are frequently counselled by motivated, supportive physicians [6, 21, 22]. However, brisk walking programmes mainly consist of endurance type exercise activities. As combined endurance and resistance type exercise training has been reported to be of greater clinical benefit $[3,23]$, it is conceivable that the long-term efficacy of a medical fitness programme is greater than that of a brisk walking programme.

In the present study, we compared the changes in glycaemic control and cardiovascular risk profile following the prescription of 12 months of either supervised group-based briskwalking or of a more individualised medical fitness programme in a large population of type 2 diabetes patients $(n=92)$ in a primary healthcare setting. We report our results for changes in $\mathrm{HbA}_{1 \mathrm{c}}$ values as a primary outcome parameter, with blood pressure, plasma lipid levels, insulin sensitivity by homeostasis model assessment (HOMA), body composition, physical fitness, programme adherence and health-related quality of life as secondary outcome parameters. Given the greater improvements in glycaemic control following combined endurance and resistance type exercise training as opposed to each separately [3, 23], we hypothesised that a medical fitness programme would result in a significantly greater decline in $\mathrm{HbA}_{1 \mathrm{c}}$ than brisk walking.

\section{Methods}

\section{Participants}

From June till December 2005, 493 patients with type 2 diabetes were preselected from a population of 12,197 patients attending a primary healthcare centre that offers structured and guideline-based [24] diabetes care programmes. After 5 months of active recruitment by two nurse practitioners and four general practitioners, a total of $76(=15.4 \%)$ patients were willing to participate in supervised exercise sessions three times a week for a period of at least 1 year. The type 2 diabetes patients selected had been diagnosed more than 3 months prior to screening according to the WHO criteria [25]. Exclusion criteria were: presence of (silent) cardiac or peripheral vascular disease, orthopaedic limitations and/or diabetic foot ulceration. To increase sample size, the exercise intervention programme was subsequently advertised in local newspapers, which yielded another 26 potential study candidates. In total, 99 type 2 diabetes patients agreed to participate in either brisk walking or medical fitness programmes. Potential study candidates performed a graded exercise test on a cycle ergometer (Medifit; Medifit Systems, Maarn, the Netherlands) using a ramp protocol [26]. Cardiac function was monitored using a 12-lead electrocardiogram and blood pressure was measured to detect malignant hypertension. Of the 99 selected patients, three did not show up and four were excluded because of an abnormal stress-ECG. The remaining 92 volunteers were randomly allocated to either brisk walking $(n=49)$ or medical fitness $(n=43)$ programmes, by subsequently drawing a sequentially numbered opaque, sealed envelope (Fig. 1). Laboratory personnel and the physician in charge of the graded exercise tests and randomisation procedure were blinded to the selection outcome of each participant. Drug prescriptions of each patient were recorded through an electronic database system operated through a network of local pharmacies. As part of the diabetes protocol in the healthcare centre, all type 2 diabetes patients consulted a dietitian and/or diabetes nurse educator annually for dietary guidance. Baseline participant characteristics are provided in Table 1. The nature and the risks of the exercise intervention were explained to all participants before written informed consent was obtained. This study was approved by the local Medical Ethical Committee of the Máxima Medical Centre, Veldhoven, the Netherlands. Since the study protocol was planned before 2005, official pre-trial registration at international trial registers was not performed.

\section{Exercise intervention}

Overview Two different exercise intervention programmes were implemented over a 12-month period, during which 


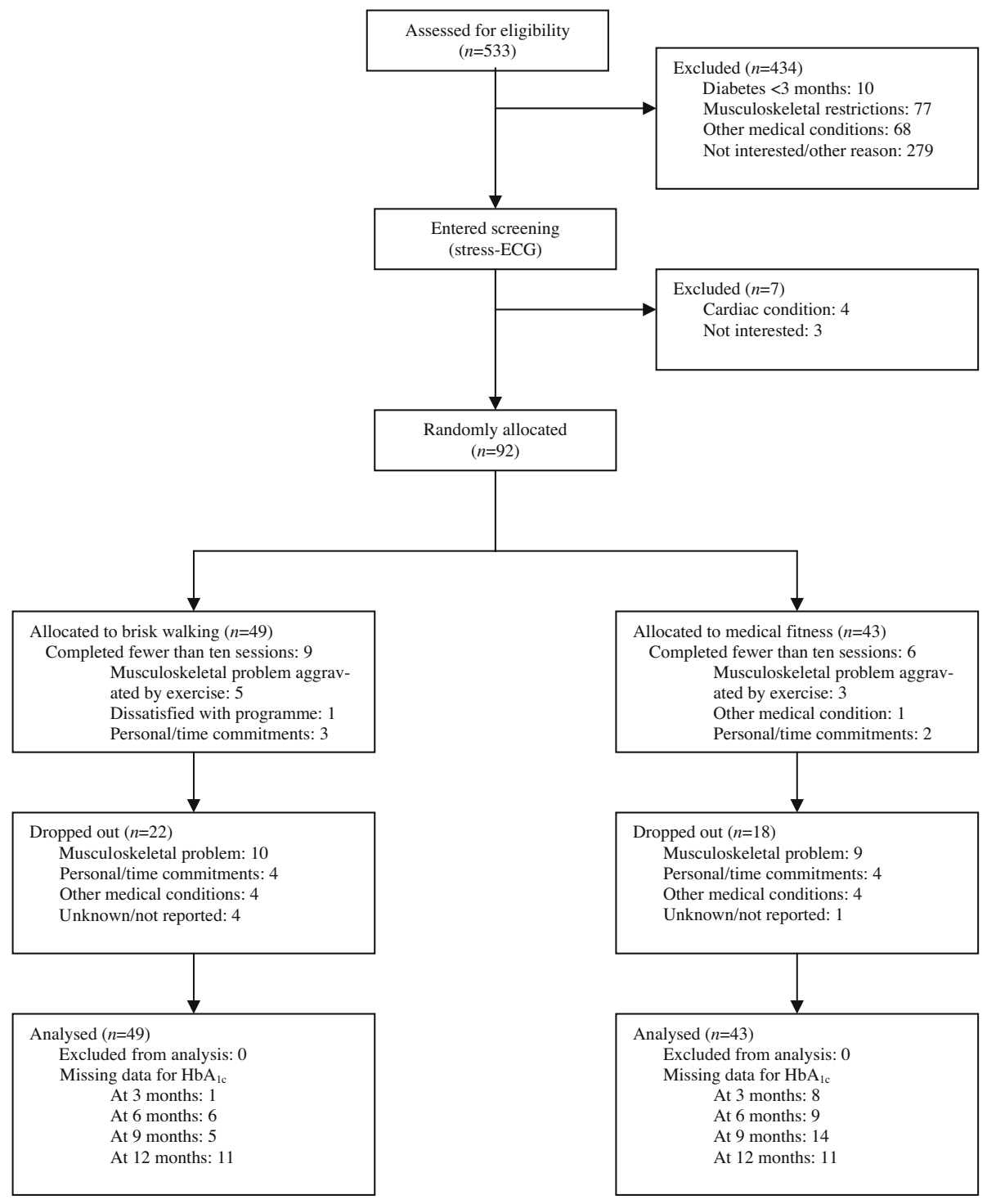

Fig. 1 Study flow diagram

three brisk walking or medical fitness sessions were performed each week. The exercise load was progressive in nature and had components of resistance and endurance type exercise according to the American Diabetes Association/ American College of Sports Medicine guidelines [27].

Brisk walking programme The weekly volume of brisk walking consisted of three 60 min exercise sessions. During the first 3 months participants were supervised by certified exercise trainers and a physical therapist. Group size varied between 15 and 25 patients. After 3 months, certified trainers guided and supervised the training sessions, while the physical therapist was visited on a consultation basis. The endurance type exercise consisted of brisk walking $(5-6 \mathrm{~km} / \mathrm{h})$, with a focus on interval type endurance exercise training. During the intervention period the intensity was gradually increased and averaged $75 \pm 5 \%$ of maximum heart frequency, as determined during the maximal cycle ergometer test. The resistance type exercise training consisted of resistance and floor exercises using individual body weight and/or elastic bands (The Hygenic Corporation, Akron, OH, USA). Further details on the brisk walking programme are provided in Electronic supplementary material (ESM) Table 1.

Medical fitness programme The medical fitness programme consisted of three exercise sessions per week. Endurance type exercise consisted of interval type exercise on a home trainer, elliptical trainer or rowing ergometer with an average intensity of $73 \pm 2 \%$ of maximum heart frequency. All training sessions were tailored to individual performance capacity. Resistance type exercise consisted of a selection of eight different exercises targeting upper and lower body muscle groups. Over a period of 6 months the training volume of the medical fitness programme was progressively increased from 
three times $30 \mathrm{~min}$ per week (90 min/week) towards a total of 180 to 225 min per week. Further details on the medical fitness programme are provided in ESM Table 1.

Energy expenditure and monitoring blood glucose Based on average heart rate and indirect calorimetry measurements performed during either brisk walking [28] or circuit resistance training in elderly [29] participants, energy expenditure in both exercise interventions was estimated to range between 0.23 and $0.33 \mathrm{~kJ} \mathrm{~kg}^{-1} \mathrm{~min}^{-1}$. Given the participants' bodyweights, the exercise interventions should be regarded as moderate intensity. Participants were provided with blood glucose monitor systems and test strips (A. Menarini Diagnostics, Valkenswaard, the Netherlands) and during the first month of brisk walking or medical fitness were advised to assess capillary blood glucose levels $\sim 30 \mathrm{~min}$ before and after exercise. Patients were instructed to report recurrent hypoglycaemic events to their diabetes nurse educator or general practitioner. For logistic reasons no systematic enquiries on mild hypoglycaemic events were performed.

Financial cost of the intervention programmes Direct yearly costs for the individualised medical fitness programme were
Euro 853 per participant and consisted of an ECG-stress test, supervision and consultations by a physical therapist, and the use of fitness centre facilities. The direct costs of the group-based brisk walking programme averaged Euro 396 per participant. Costs consisted of an ECG-stress test, supervision and consultation by a physical therapist, and a brisk walking membership fee. Other healthcare-related costs were not assessed.

Outcome variables

Resting heart rate and blood pressure Resting heart rate and blood pressure were determined in a supine position before and at 6 and 12 months after initiating the exercise programme. Heart rate was stored and averaged through a heart rate monitoring system (CO2ntrol; Tildesign, Zeewolde, the Netherlands). Average systolic and diastolic blood pressure were determined (HEM907; Omron Healthcare, Hoofddorp, the Netherlands) from five successive measurements.

Physical fitness Before and at 12 months after the start of the exercise programme, peak oxygen uptake capacity $\left(\dot{V} \mathrm{O}_{2 \text { peak }}\right)$ was estimated based on maximum workload capacity $\left(W_{\max }\right)$
Table 1 Participants' baseline characteristics
Data are $n$ (number of participants) or means $\pm \mathrm{SD}$.

Estimated $\dot{V} \mathrm{O}_{2 \text { peak }}$ was based on $W_{\max }$ during cycling ergometry and the equation according to Storer et al. [30]. Relative age-, height- and sex-adjusted cardio-respiratory fitness (\% \%red. $\dot{V} \mathrm{O}_{2 \text { peak }}$ ) was based on the equation by Fairbarn et al. [31].

ACEI, ACE inhibitor; ARB, angiotensin receptor blocker; BW, body weight

\begin{tabular}{|c|c|c|c|}
\hline Characteristic & Total $(n=92)$ & Brisk walking $(n=49)$ & Medical fitness $(n=43)$ \\
\hline Age (years) & $60 \pm 9$ & $61 \pm 9$ & $59 \pm 9$ \\
\hline Men/women $(n)$ & $47 / 45$ & $27 / 22$ & $20 / 23$ \\
\hline Duration of diabetes (years) & $5 \pm 5$ & $5 \pm 4$ & $5 \pm 5$ \\
\hline BW (kg) & $94.3 \pm 18.4$ & $94.1 \pm 18.1$ & $94.5 \pm 19.0$ \\
\hline BMI $\left(\mathrm{kg} / \mathrm{m}^{2}\right)$ & $32.3 \pm 5.5$ & $32.1 \pm 5.2$ & $32.5 \pm 6.0$ \\
\hline$W_{\max }(\mathrm{W})$ & $156 \pm 47$ & $155 \pm 49$ & $157 \pm 45$ \\
\hline Estimated $\dot{V} \mathrm{O}_{2 \text { peak }}(1 / \mathrm{min})$ & $2.1 \pm 0.6$ & $2.1 \pm 0.6$ & $2.1 \pm 0.6$ \\
\hline$\%$ Pred. $\dot{V} \mathrm{O}_{2 \text { peak }}$ & $82 \pm 11$ & $82 \pm 10$ & $83 \pm 11$ \\
\hline \multicolumn{4}{|l|}{ Participants treated with: } \\
\hline Diet only $(n)$ & 11 & 5 & 6 \\
\hline \multicolumn{4}{|l|}{ Hypoglycaemic agents $(n)$} \\
\hline Total & 81 & 44 & 37 \\
\hline Metformin & 43 & 24 & 19 \\
\hline Sulfonylurea & 25 & 13 & 12 \\
\hline Thiazolidinedione & 10 & 4 & 6 \\
\hline Insulin & 13 & 6 & 7 \\
\hline \multicolumn{4}{|l|}{ Antihypertensive agents $(n)$} \\
\hline Total & 59 & 32 & 27 \\
\hline ACEI/ARB & 39 & 20 & 19 \\
\hline Beta-blocker & 17 & 7 & 10 \\
\hline Diuretic & 19 & 10 & 9 \\
\hline Calcium-channel blocker & 9 & 5 & 4 \\
\hline Other & 3 & 1 & 2 \\
\hline \multicolumn{4}{|l|}{ Lipid-lowering agents $(n)$} \\
\hline Total & 54 & 30 & 24 \\
\hline Statin & 53 & 29 & 24 \\
\hline Fibrate & 1 & 1 & 0 \\
\hline Other & 0 & 0 & 0 \\
\hline
\end{tabular}


during cycling ergometry according to Storer et al. [30]. Relative age-, height- and sex-adjusted cardio-respiratory fitness (\%Pred. $\dot{V} \mathrm{O}_{2 \text { peak }}$ ) was defined as the ratio between estimated $\dot{V} \mathrm{O}_{2 \text { peak }}$ and predicted $\dot{V} \mathrm{O}_{2 \text { peak }}$ as determined in a healthy non-diabetic population [31].

Blood analyses Two weeks before and at 3, 6, 9 and 12 months after initiating the exercise programme, fasting blood samples were collected. Blood samples collected before and after 3 and 9 months of intervention were analysed for $\mathrm{HbA}_{1 \mathrm{c}}$ and basal glucose concentration. On the evening prior to blood sampling, participants remained fasted from 00:00 hours onwards. They arrived at the laboratory at 08:00 hours, having travelled by car or public transport. After 5 to $10 \mathrm{~min}$ of rest, a venous blood sample was collected from an antecubital vein. Blood samples were collected in tubes containing a glycolytic inhibitor (sodium fluoride) and an anticoagulant (potassium oxalate), immediately centrifuged for $5 \mathrm{~min}$ at $1,000 \times \mathrm{g}$ and $4^{\circ} \mathrm{C}$, after which aliquots of plasma were immediately frozen in liquid nitrogen and stored at $-80^{\circ} \mathrm{C}$ until analyses. Plasma glucose (dehydrogenase assay), triacylglycerol (lipase/peroxidase assay) concentrations, and total serum cholesterol (peroxidase/cholesterolesterase assay), HDL-cholesterol (peroxidase esterase assay) and LDL-cholesterol (according to the Friedewald formula if triacylglycerol was $<4.5 \mathrm{mmol} / \mathrm{l}$, otherwise through direct peroxidase esterase assay) were analysed (Synchron LX20; Beckman Coulter, Fullerton, CA, USA). Plasma insulin was determined in duplicate by electrochemiluminescenceimmunoassay (Elecsys 2010; Roche, Mannheim, Germany). Because of cross-sensitivity in the latter assay, exogenous insulin users were excluded from this analysis. HOMA insulin resistance index [32] was assessed to monitor changes in insulin sensitivity [33]. To determine $\mathrm{HbA}_{1 \mathrm{c}}$, $3 \mathrm{ml}$ blood samples were collected and analysed by highperformance liquid chromatography (HA8160 Menarini; A. Menarini Diagnostics, Florence, Italy).

\section{Quality-of-life assessment}

Health-related quality-of-life was measured with the RAND 36-Item Health Survey $1.0[34,35]$ before and after 12 months of exercise intervention.

\section{Statistical analyses}

We calculated that 74 persons ( 37 per group) were needed to have $80 \%$ power to detect a moderate 0.65 SD difference in $\mathrm{HbA}_{1 \mathrm{c}}$ as primary endpoint parameter between brisk walking and medical fitness programme, with an $\alpha$ of 0.05. Sample size was exceeded to allow for an estimated withdrawal of $\sim 15 \%$. Data were analysed according to the intention-to-treat (ITT) principle. To minimise type I errors and loss of power
[36], endpoint analyses according to the last observation carried forward principle were performed for missing values at 12 months follow-up. To assess whether physiological differences existed between the long-term application of brisk walking and medical fitness programme, post hoc analyses were performed for individuals actively participating for 10 months or more. Data are expressed as means \pm SD. ANOVA repeated measures was used to determine differences between baseline and status after 12 months of exercise intervention. Unpaired Student's $t, \chi^{2}$ and MannWhitney $U$ tests were used to test whether long-term changes observed in brisk walking differed from those seen in the medical fitness programme. Significance was set at the 0.05 level of confidence. Pearson's correlation calculation was used to test for linear relationships between long-term changes in dependent variables. All statistical calculations were performed using SPSS 10.1 (SPSS, Chicago, IL, USA).

\section{Results}

Characteristics of the study population

Following randomisation there were no significant differences in baseline characteristics, sex and anthropometry between brisk walking and medical fitness programme groups (Table 1).

Programme adherence, follow-up and adverse events

After 6 months of intervention, 22 (45\%) brisk walking participants and $13(30 \%)$ medical fitness programme participants $(p=0.15$ ) were no longer participating (ESM Table 2). After 12 months, 18 (37\%) brisk walking and 19 (44\%) medical fitness participants respectively were still actively participating with mean adherence levels of $75 \pm 16 \%$ and $68 \pm 13 \%(p>0.05)$ for the 156 available exercise sessions (ESM Table 2). Besides motivational reasons (25\%), orthopaedic-related co-morbidities, such as overuse injuries and/or subclinical osteoarthritis of the lower extremities, formed the main reason for dropout in 48 and $50 \%$ of the brisk walking and medical fitness programme participants, respectively (Fig. 1, Table 2). The distribution of missing data points was similar and not statistically different between groups $(p>0.05)$.

\section{Glycaemic control}

According to ITT analyses, changes in $\mathrm{HbA}_{1 \mathrm{c}}$ values following the prescription of brisk walking or medical fitness intervention were identical $(95 \%$ CI $-0.42,0.43$; $p=0.99$ ) (Table 3). A total of 12 patients (brisk walking, 
$n=5$; medical fitness, $n=7$ ) had been prescribed higher doses of blood glucose-lowering medication throughout the follow-up period. When these participants were excluded from our ITT analysis, $\mathrm{HbA}_{1 \mathrm{c}}$ increased by $0.05 \%(95 \%$ CI $-0.41,0.51 ; p=0.82$ ) in brisk walking as compared with medical fitness programme. Post hoc analyses of long-term active participants $(\geq 10$ months with mean adherence level of $62 \pm 17$ and $70 \pm 18 \%$ in brisk walking and medical fitness groups, respectively) showed that, independently of changes in prescribed blood glucose-lowering drugs, $\mathrm{HbA}_{1 \mathrm{c}}$ in the brisk walking group increased by $0.53 \%$ (95\% CI 0.07 , $1.00 ; p=0.025$ ) as compared with the medical fitness group (ESM Table 3).

Resting heart rate and blood pressure

Following the 12 month exercise intervention, resting heart rate in the brisk walking group was reduced by 0.9 beats per $\min (\mathrm{bpm})(\mathrm{CI}-7.1,5.3 ; p=0.68)$ as compared with medical fitness group. No significant differences in blood pressure response were observed between the two interventions (Table 3). After excluding participants who were prescribed higher doses of antihypertensive drugs (brisk walking, $n=5$; medical fitness, $n=7$ ), mean arterial blood pressure decreased by $0.9 \mathrm{mmHg}(95 \% \mathrm{CI}-5.6,3.9 ; p=0.94)$ in the brisk walking as compared with medical fitness group. A post hoc analysis of 46 long-term active participants showed a non-significant difference in mean arterial blood pressure of $-3.5 \mathrm{mmHg}(95 \% \mathrm{CI}-9.2,2.2 ; p=0.22)$ in the brisk walking compared with the medical fitness programme (ESM Table 3).
Blood lipid profile

After 12 months, the overall fasting lipid profile did not differ between the two groups (Table 3). When participants (brisk walking, $n=9$, medical fitness, $n=7$ ) who were prescribed more blood lipid-lowering agents were excluded, HDLcholesterol had changed by $-0.05 \mathrm{mmol} / 1(95 \%$ CI -0.12 , $0.02 ; p=0.17$ ) in the medical fitness compared with the brisk walking group.

Body composition and workload capacity

Body composition (BMI) did not change (Table 3). Workload capacity as measured during cycle ergometry averaged $155 \pm$ 49 and $157 \pm 45 \mathrm{~W}$ in the brisk walking and medical fitness programme groups, respectively, changing by $-4.3 \mathrm{~W}(95 \%$ CI $-9.1,0.5 ; p=0.078)$ in the former and by $-2.5 \mathrm{~W}(95 \%$ $\mathrm{CI}-10,5.6 ; p=0.53)$ in the latter, with a non-significant difference of $-1.8 \mathrm{~W}(95 \% \mathrm{CI}-10.5,6.9 ; p=0.68)$ in brisk walking compared with medical fitness programme participants. Maximum heart rates recorded during ergometry averaged $146 \pm 22$ and $152 \pm 19 \mathrm{bpm}$ at baseline, and $152 \pm 21$ and $149 \pm 22 \mathrm{bpm}$ following 12 months of exercise intervention in the brisk walking and medical fitness groups, respectively $(p>0.05)$. In our long-term active brisk walking participants, $W_{\max }$ changed by $-6.3 \mathrm{~W}(95 \% \mathrm{CI}-16.5,3.9$; $p=0.22$ ) as compared with their medical fitness programme counterparts. No significant correlations were found between changes in $\mathrm{HbA}_{1 \mathrm{c}}$ values and changes in workload capacity ( $\Delta W_{\max }$, Pearson's $R=0.02, p=0.93, n=38$ ) or body weight (Pearson's $R=0.18, p=0.225, n=45$ ).

Table 2 Adverse medical events

\begin{tabular}{|c|c|c|}
\hline Events & Brisk walking $(n=49)$ & Medical fitness $(n=43)$ \\
\hline Adverse events related to exercise intervention & 15 & 12 \\
\hline Shoulder pain/chronic tendinopathy of rotator cuff & 0 & 1 \\
\hline (Aggravation of) low back pain & 2 & 2 \\
\hline Aggravation of pre-existing osteoarthritis in hip or knee joint & 6 & 4 \\
\hline Shin splints/lower leg pain & 1 & 0 \\
\hline Chronic tendinopathy of Achilles tendon/plantar fascia & 3 & 1 \\
\hline Other/generalised musculoskeletal discomfort & 3 & 3 \\
\hline Adverse events not related to exercise intervention & 4 & 6 \\
\hline Medical event requiring hospitalisation ${ }^{\mathrm{a}}$ & 3 & 3 \\
\hline Other serious medical event ${ }^{\mathrm{b}}$ & 1 & 3 \\
\hline Participants with adverse event resulting in withdrawal & 19 & 17 \\
\hline
\end{tabular}

Data are number of participating diabetes patients that experienced an adverse event throughout the 12 months follow-up period

${ }^{\text {a }}$ Hospitalisations were related to: arthroscopic knee surgery (brisk walking, $n=1$ ), elective surgery for chronic abdominal aortic aneurysm (brisk walking, $n=1$ ), elective cataract surgery (brisk walking, $n=1$ ), elective knee joint replacement (medical fitness, $n=1$ ), bacterial pneumonia (medical fitness, $n=1$ ), elective varicose veins surgery (medical fitness, $n=1$, did not result in withdrawal from study).

${ }^{\mathrm{b}}$ Other serious medical events requiring medical attention were: otitis externa (brisk walking) $n=1$, atrial fibrillation (medical fitness) $n=1$, newly diagnosed myocardial ischaemia (medical fitness) $n=1$, and newly diagnosed carcinoma of the mammary gland (medical fitness) $n=1$ 
Table 3 Changes in diabetes outcome (ITT analysis)

\begin{tabular}{|c|c|c|c|c|c|c|c|}
\hline \multirow[t]{2}{*}{ Variable } & \multicolumn{7}{|c|}{ Change from baseline to 12 months } \\
\hline & Mean \pm SD & $n$ & Mean $\pm \mathrm{SD}$ & $n$ & Difference & $95 \% \mathrm{CI}$ & $p$ Value \\
\hline \multicolumn{8}{|l|}{$\mathrm{HbA}_{1 \mathrm{c}}(\%)$} \\
\hline Total & $7.13 \pm 1.36$ & 92 & $6.99 \pm 1.26$ & 71 & & & \\
\hline Brisk walking group & $7.18 \pm 1.42$ & 49 & $7.08 \pm 1.37$ & 38 & & & \\
\hline Medical fitness group & $7.08 \pm 1.29$ & 43 & $6.89 \pm 1.13$ & 33 & & & \\
\hline \multicolumn{8}{|l|}{ Intragroup comparisons } \\
\hline Intragroup comparison total & & & & & -0.14 & $-0.39,0.03$ & 0.153 \\
\hline Intragroup comparison brisk walking & & & & & -0.11 & $-0.51,0.15$ & 0.464 \\
\hline Intragroup comparison medical fitness & & & & & -0.18 & $-0.45,0.09$ & 0.176 \\
\hline Brisk walking vs medical fitness & & & & & 0.00 & $-0.42,0.43$ & 0.985 \\
\hline \multicolumn{8}{|l|}{ BMI $\left(\mathrm{kg} / \mathrm{m}^{2}\right)$} \\
\hline Total & $32.3 \pm 5.5$ & 92 & $31.8 \pm 5.4$ & 89 & & & \\
\hline Brisk walking group & $32.1 \pm 5.2$ & 49 & $31.9 \pm 5.0$ & 48 & & & \\
\hline Medical fitness group & $32.5 \pm 6.0$ & 43 & $31.7 \pm 5.9$ & 41 & & & \\
\hline \multicolumn{8}{|l|}{ Intragroup comparisons } \\
\hline Intragroup comparison total & & & & & -0.5 & $-1.1,0.1$ & 0.108 \\
\hline Intragroup comparison brisk walking & & & & & -0.2 & $-0.6,0.3$ & 0.471 \\
\hline Intragroup comparison medical fitness & & & & & -0.8 & $-2.0,0.3$ & 0.152 \\
\hline Brisk walking vs medical fitness & & & & & 0.7 & $-0.5,1.9$ & 0.237 \\
\hline \multicolumn{8}{|l|}{ Fasting plasma glucose $(\mathrm{mmol} / \mathrm{l})$} \\
\hline Total & $8.44 \pm 2.81$ & 92 & $8.21 \pm 2.37$ & 71 & & & \\
\hline Brisk walking group & $8.64 \pm 2.83$ & 49 & $8.33 \pm 2.64$ & 38 & & & \\
\hline Medical fitness group & $8.21 \pm 2.80$ & 43 & $8.06 \pm 2.05$ & 33 & & & \\
\hline \multicolumn{8}{|l|}{ Intragroup comparisons } \\
\hline Intragroup comparison total & & & & & -0.23 & $-0.72,0.25$ & 0.345 \\
\hline Intragroup comparison brisk walking & & & & & -0.31 & $-0.96,0.34$ & 0.344 \\
\hline Intragroup comparison medical fitness & & & & & -0.15 & $-0.91,0.61$ & 0.699 \\
\hline Brisk walking vs. medical fitness & & & & & -0.16 & $-1.14,0.82$ & 0.744 \\
\hline \multicolumn{8}{|l|}{$\mathrm{HOMA}^{\mathrm{a}}$} \\
\hline Total & $5.55 \pm 3.68$ & 68 & $5.60 \pm 3.38$ & 54 & & & \\
\hline Brisk walking group & $5.86 \pm 3.56$ & 37 & $5.75 \pm 3.20$ & 30 & & & \\
\hline Medical fitness group & $5.19 \pm 3.58$ & 31 & $5.43 \pm 3.64$ & 24 & & & \\
\hline \multicolumn{8}{|l|}{ Intragroup comparisons } \\
\hline Intragroup comparison total & & & & & 0.05 & $-0.50,0.62$ & 0.833 \\
\hline Intragroup comparison brisk walking & & & & & -0.12 & $-1.11,0.85$ & 0.794 \\
\hline Intragroup comparison medical fitness & & & & & 0.24 & $-0.19,0.74$ & 0.241 \\
\hline Brisk walking vs medical fitness & & & & & -0.40 & $-1.52,0.72$ & 0.479 \\
\hline \multicolumn{8}{|l|}{ Resting heart rate (bpm) } \\
\hline Total & $73.4 \pm 11.8$ & 91 & $69.6 \pm 13.9$ & 77 & & & \\
\hline Brisk walking group & $72.6 \pm 11.8$ & 48 & $68.3 \pm 15.0$ & 41 & & & \\
\hline Medical fitness group & $74.4 \pm 11.8$ & 43 & $71.0 \pm 12.4$ & 36 & & & \\
\hline \multicolumn{8}{|l|}{ Intragroup comparisons } \\
\hline Intragroup comparison total & & & & & -3.9 & $-5.9,-1.8$ & 0.000 \\
\hline Intragroup comparison brisk walking & & & & & -4.3 & $-7.4,-1.1$ & 0.009 \\
\hline Intragroup comparison medical fitness & & & & & -3.4 & $-5.9,-0.9$ & 0.010 \\
\hline Brisk walking vs medical fitness & & & & & -0.9 & $-4.9,3.2$ & 0.678 \\
\hline \multicolumn{8}{|l|}{ Systolic blood pressure (mmHg) } \\
\hline Total & $148.5 \pm 18.1$ & 91 & $137.5 \pm 15.5$ & 77 & & & \\
\hline Brisk walking group & $150.3 \pm 18.9$ & 48 & $138.9 \pm 16.3$ & 41 & & & \\
\hline Medical fitness group & $146.3 \pm 17.1$ & 43 & $135.9 \pm 14.6$ & 36 & & & \\
\hline \multicolumn{8}{|l|}{ Intragroup comparisons } \\
\hline Intragroup comparison total & & & & & -10.9 & $-14.0,-7.8$ & 0.000 \\
\hline Intragroup comparison brisk walking & & & & & -11.4 & $-15.9,-6.8$ & 0.000 \\
\hline Intragroup comparison medical fitness & & & & & -10.4 & $-14.7,6.2$ & 0.000 \\
\hline Brisk walking vs medical fitness & & & & & -0.9 & $-7.1,5.3)$ & 0.768 \\
\hline
\end{tabular}


Table 3 (continued)

\begin{tabular}{|c|c|c|c|c|c|c|c|}
\hline \multirow[t]{2}{*}{ Variable } & \multicolumn{7}{|c|}{ Change from baseline to 12 months } \\
\hline & Mean \pm SD & $n$ & Mean \pm SD & $n$ & Difference & $95 \% \mathrm{CI}$ & $p$ Value \\
\hline \multicolumn{8}{|l|}{ Diastolic blood pressure $(\mathrm{mmHg})$} \\
\hline Total & $81.9 \pm 10.6$ & 91 & $76.6 \pm 8.3$ & 77 & & & \\
\hline Brisk walking group & $81.3 \pm 10.8$ & 48 & $75.9 \pm 8.8$ & 41 & & & \\
\hline Medical fitness group & $82.6 \pm 10.4$ & 43 & $77.4 \pm 7.7$ & 36 & & & \\
\hline \multicolumn{8}{|l|}{ Intragroup comparisons } \\
\hline Intragroup comparison total & & & & & -5.3 & $-7.1,-3.6$ & 0.000 \\
\hline Intragroup comparison brisk walking & & & & & -5.4 & $-7.8,2.9$ & 0.000 \\
\hline Intragroup comparison medical fitness & & & & & -5.2 & $-7.8,-2.7$ & 0.000 \\
\hline Brisk walking vs medical fitness & & & & & -0.1 & $-3.6,3.4$ & 0.949 \\
\hline \multicolumn{8}{|l|}{ Total cholesterol level $(\mathrm{mmol} / \mathrm{l})$} \\
\hline Total & $4.70 \pm 0.86$ & 88 & $4.53 \pm 0.94$ & 65 & & & \\
\hline Brisk walking group & $4.83 \pm 0.89$ & 47 & $4.61 \pm 0.92$ & 35 & & & \\
\hline Medical fitness group & $4.54 \pm 0.12$ & 41 & $4.44 \pm 0.96$ & 30 & & & \\
\hline \multicolumn{8}{|l|}{ Intragroup comparisons } \\
\hline Intragroup comparison total & & & & & -0.16 & $-0.32,-0.01$ & 0.035 \\
\hline Intragroup comparison brisk walking & & & & & -0.22 & $-0.45,0.01$ & 0.064 \\
\hline Intragroup comparison medical fitness & & & & & -0.10 & $-0.30,0.10$ & 0.307 \\
\hline Brisk walking vs medical fitness & & & & & -0.12 & $-0.42,0.19$ & 0.453 \\
\hline \multicolumn{8}{|l|}{ LDL-cholesterol level $(\mathrm{mmol} / \mathrm{l})$} \\
\hline Total & $2.83 \pm 0.76$ & 88 & $2.70 \pm 0.79$ & 64 & & & \\
\hline Brisk walking group & $2.90 \pm 0.82$ & 47 & $2.72 \pm 0.85$ & 35 & & & \\
\hline Medical fitness group & $2.74 \pm 0.69$ & 41 & $2.68 \pm 0.72$ & 29 & & & \\
\hline \multicolumn{8}{|l|}{ Intragroup comparisons } \\
\hline Intragroup comparison total & & & & & -0.12 & $-0.26,0.01$ & 0.070 \\
\hline Intragroup comparison brisk walking & & & & & -0.18 & $-0.38,0.02$ & 0.080 \\
\hline Intragroup comparison medical fitness & & & & & -0.06 & $-0.25,0.12$ & 0.493 \\
\hline Brisk walking vs medical fitness & & & & & -0.11 & $-0.38,0.16$ & 0.401 \\
\hline \multicolumn{8}{|l|}{ HDL-cholesterol level $(\mathrm{mmol} / \mathrm{l})$} \\
\hline Total & $1.09 \pm 0.28$ & 88 & $1.10 \pm 0.29$ & 65 & & & \\
\hline Brisk walking group & $1.10 \pm 0.23$ & 47 & $1.09 \pm 0.26$ & 35 & & & \\
\hline Medical fitness group & $1.07 \pm 0.33$ & 41 & $1.11 \pm 0.32$ & 30 & & & \\
\hline \multicolumn{8}{|l|}{ Intragroup comparisons } \\
\hline Intragroup comparison total & & & & & -0.01 & $-0.02,0.04$ & 0.570 \\
\hline Intragroup comparison brisk walking & & & & & -0.01 & $-0.06,0.04$ & 0.629 \\
\hline Intragroup comparison medical fitness & & & & & 0.03 & $-0.01,0.08$ & 0.129 \\
\hline Brisk walking vs medical fitness & & & & & -0.05 & $-0.12,0.02$ & 0.172 \\
\hline \multicolumn{8}{|l|}{ Triacylglycerol level (mmol/l) } \\
\hline Total & $1.88 \pm 0.99$ & 88 & $1.79 \pm 0.91$ & 64 & & & \\
\hline Brisk walking group & $2.00 \pm 1.08$ & 47 & $1.94 \pm 0.98$ & 35 & & & \\
\hline Medical fitness group & $1.74 \pm 0.88$ & 41 & $1.61 \pm 0.79$ & 29 & & & \\
\hline \multicolumn{8}{|l|}{ Intragroup comparisons } \\
\hline Intragroup comparison total & & & & & -0.09 & $-0.25,0.06$ & 0.239 \\
\hline Intragroup comparison brisk walking & & & & & -0.06 & $-0.33,0.17$ & 0.646 \\
\hline Intragroup comparison medical fitness & & & & & -0.13 & $-0.27,0.06$ & 0.120 \\
\hline Brisk walking vs medical fitness & & & & & -0.02 & $-0.29,0.33$ & 0.892 \\
\hline \multicolumn{8}{|l|}{ RAND-36 (score 0-100 scale) } \\
\hline Total & $71 \pm 15$ & 89 & $70 \pm 15$ & 58 & & & \\
\hline Brisk walking group & $69 \pm 15$ & 46 & $68 \pm 15$ & 31 & & & \\
\hline Medical fitness group & $73 \pm 15$ & 43 & $71 \pm 14$ & 27 & & & \\
\hline \multicolumn{8}{|l|}{ Intragroup comparisons } \\
\hline Intragroup comparison total & & & & & -0.6 & $-2.4,1.2$ & 0.503 \\
\hline Intragroup comparison brisk walking & & & & & 0.1 & $-2.7,2.8$ & 0.969 \\
\hline Intragroup comparison medical fitness & & & & & -1.4 & $-3.8,1.1$ & 0.261 \\
\hline Brisk walking vs medical fitness & & & & & 1.4 & $-2.2,5.0$ & 0.438 \\
\hline
\end{tabular}

Data are means \pm SD. $n=$ number of valid measurements. ${ }^{\text {a }}$ For HOMA calculations exogenous insulin users $(n=13)$ were excluded 
Quality of life assessment

A total of 89 and 58 participants completed the RAND-36 questionnaire at baseline and after 12 months of intervention, respectively. Reliability and internal consistency was excellent with Cronbach's alpha of 0.93 and 0.91 respectively. No significant difference in changes of total RAND36 scores were observed between the two intervention groups ( $p>0.05$; Table 3$)$.

\section{Discussion}

It has been firmly established that physical activity counselling [37] and participation in structured exercise intervention programmes $[15,19]$ improves glycaemic control. However, long-term intervention studies on the clinical benefits of different types of exercise intervention programmes in type 2 diabetes patients are lacking. In the present study, we compared the clinical benefits of 12 months of a groupbased brisk walking programme versus a more individualised medical fitness programme. ITT analysis showed that the prescription of a group-based, brisk walking programme is not necessarily inferior to a more individualised medical fitness programme with regard to glycaemic control and markers for cardiovascular risk profile (Table 3). Our data therefore imply that most patients with type 2 diabetes will achieve more or less equal long-term therapeutic benefits from a low-impact brisk walking programme as opposed to a more individualised medical fitness programme. Nevertheless, long-term programme adherence and the possibility of dropout should be taken into consideration when prescribing either type of exercise intervention for a patient with type 2 diabetes. Independently of the provided level of guidance and infrastructure, $60 \%$ of the patients dropped out of the exercise programme during the 12 month intervention period, with no differences in adherence and dropout patterns between brisk walking and medical fitness programme. Although motivational factors explained $25 \%$ of the dropout in both exercise programmes, almost $50 \%$ of the dropout was attributed to orthopaedic-related comorbidities and overuse injuries of the lower extremities. The latter was not anticipated, since clinically relevant orthopaedic limitations, such as a history of osteoarthritis of the hip or knee joint, were defined as exclusion criteria for participation in either exercise intervention programme. Apparently, subclinical joint disease and/or degeneration of myotendineous structures become apparent when physical activity levels are increased. Stiffening of connective tissue due to non-enzymatic advanced glycation end-product formation [38], as well as decreased collagen turnover due to ageing and chronic disuse [39], could be responsible for the impaired response to increased musculoskeletal loading in these patients. Although more fundamental clinical research is warranted, the high incidence of overuse injuries in the present study adds to the results described in another study [23], suggesting that in unselected, non-study populations dropout will be even greater. Therefore, it might be advisable to implement a more gradual and less intense exercise regimen to allow myotendineous structures to adapt to increased loading. Moreover, before prescribing therapeutic exercise, diabetes healthcare workers should carefully consider obesity- and diabetes-related musculoskeletal deconditioning [40-42]. Such programmes should probably focus even more on resistance type exercise activities [43, 44] to bring patients to a level at which they are able to participate and adhere to more generic diabetes intervention programmes. It has also been suggested that psychological strategies such as motivational interviewing [45] or booster sessions $[21,46]$ might help to further improve programme adherence. Nevertheless, more long-term, tailored exercise intervention studies are warranted to further define the most effective and feasible exercise interventional strategy.

The absence of a significant decline in blood $\mathrm{HbA}_{1 \mathrm{c}}$ levels in brisk walking participants does not imply that the prescription of low-impact, endurance type exercise has no therapeutic value $[3,7,27,47-49]$. In fact, we observed significant improvements in blood pressure control following either type of exercise intervention (Table 3). Although reliable assessment of actual drug use was not possible in the present study, the improvements in blood pressure occurred independently of changes in the prescription of antihypertensive medication. However, the lack of a non-exercise control group in the present study makes it impossible to speculate on cause and effect, since changes in blood pressure from baseline might also, or at least partly, be explained by the Hawthorne effect, for example, or by familiarisation with blood pressure measurement protocols. Nevertheless, our data suggest that the structured application of group-based brisk walking and supervised medical fitness programmes tends to have a similar impact on cardiovascular risk profile.

In conclusion, the prescription of group-based brisk walking represents an equally effective interventional strategy to modulate glycaemic control and cardiovascular risk profile in type 2 diabetes patients compared with a more individualised medical fitness programme. General deconditioning, musculoskeletal overuse injuries and lack of motivation limit the benefits of long-term exercise intervention as an add-on to a comprehensive diabetes care programme. Future diabetes exercise intervention programmes in primary healthcare settings should consider diabetes-related comorbidities and patient motivation as important factors, which determine both long-term programme adherence and the associated clinical benefits. 
Acknowledgements This study was funded by a Dutch Healthcare Innovation Foundation research grant from 'OZ-zorgverzekeringen' healthcare insurance company. The salary of S. F. Praet was funded by a research grant from the Dutch Ministry of Health, Welfare and Sports. The authors thank all participating participants, as well as M. van Hoogstraten and all certified trainers from AVR Achilles and BodyActive for participating in and supervising the exercise programmes, respectively. A. Menarini Diagnostics Benelux supplied our patients with free blood glucose monitoring systems and test strips. RSscan International provided our participants with free protective footwear and customised insoles. We are also extremely grateful to S. Konings, J. Lambregts, H. Wierema, M. Schaerlaeckens, A. van der Smissen, R. Lendfers, R. Swens, R. van Hal, S. van der Poel, R. Masseurs, H. Bronts, P. Litjens, S. van Rooij and L. van den Meijdenberg for their input and logistic support.

Duality of interest The authors declare that there is no duality of interest associated with this study.

Open Access This article is distributed under the terms of the Creative Commons Attribution Noncommercial License which permits any noncommercial use, distribution, and reproduction in any medium, provided the original author(s) and source are credited.

\section{References}

1. Berger M (1976) Muscular work in the therapy of diabetes mellitus. Fortschr Med 94:1553-1557

2. Knowler WC, Barrett-Connor E, Fowler SE et al (2002) Reduction in the incidence of type 2 diabetes with lifestyle intervention or metformin. N Engl J Med 346:393-403

3. Snowling NJ, Hopkins WG (2006) Effects of different modes of exercise training on glucose control and risk factors for complications in type 2 diabetic patients: a meta-analysis. Diabetes Care 29:2518-2527

4. Thomas DE, Elliott EJ, Naughton GA (2006) Exercise for type 2 diabetes mellitus. Cochrane Database Syst Rev 3:CD002968

5. Conn VS, Hafdahl AR, Mehr DR, Lemaster JW, Brown SA, Nielsen PJ (2007) Metabolic effects of interventions to increase exercise in adults with type 2 diabetes. Diabetologia 50:913-921

6. Di Loreto C, Fanelli C, Lucidi P et al (2005) Make your diabetic patients walk: long-term impact of different amounts of physical activity on type 2 diabetes. Diabetes Care 28:1295-1302

7. Boule NG, Kenny GP, Haddad E, Wells GA, Sigal RJ (2003) Metaanalysis of the effect of structured exercise training on cardiorespiratory fitness in Type 2 diabetes mellitus. Diabetologia 46:10711081

8. Nielsen PJ, Hafdahl AR, Conn VS, Lemaster JW, Brown SA (2006) Meta-analysis of the effect of exercise interventions on fitness outcomes among adults with type 1 and type 2 diabetes. Diabetes Res Clin Pract 74:111-120

9. Boule NG, Haddad E, Kenny GP, Wells GA, Sigal RJ (2001) Effects of exercise on glycemic control and body mass in type 2 diabetes mellitus: a meta-analysis of controlled clinical trials. Jama 286:1218-1227

10. Burns N, Finucane FM, Hatunic M et al (2007) Early-onset type 2 diabetes in obese white subjects is characterised by a marked defect in beta cell insulin secretion, severe insulin resistance and a lack of response to aerobic exercise training. Diabetologia 50:1500-1508

11. Dunstan DW, Vulikh E, Owen N, Jolley D, Shaw J, Zimmet P (2006) Community center-based resistance training for the main- tenance of glycemic control in adults with type 2 diabetes. Diabetes Care 29:2586-2591

12. Tokmakidis SP, Zois CE, Volaklis KA, Kotsa K, Touvra AM (2004) The effects of a combined strength and aerobic exercise program on glucose control and insulin action in women with type 2 diabetes. Eur J Appl Physiol 92:437-442

13. McGavock J, Mandic S, Lewanczuk R et al (2004) Cardiovascular adaptations to exercise training in postmenopausal women with type 2 diabetes mellitus. Cardiovasc Diabetol 3:3

14. Alam S, Stolinski M, Pentecost C et al (2004) The effect of a sixmonth exercise program on very low-density lipoprotein apolipoprotein B secretion in type 2 diabetes. J Clin Endocrinol Metab 89:688-694

15. Loimaala A, Huikuri HV, Koobi T, Rinne M, Nenonen A, Vuori I (2003) Exercise training improves baroreflex sensitivity in type 2 diabetes. Diabetes 52:1837-1842

16. Castaneda C, Layne JE, Munoz-Orians L et al (2002) A randomised controlled trial of resistance exercise training to improve glycemic control in older adults with type 2 diabetes. Diabetes Care 25:2335-2341

17. Ligtenberg PC, Hoekstra JB, Bol E, Zonderland ML, Erkelens DW (1997) Effects of physical training on metabolic control in elderly type 2 diabetes mellitus patients. Clin Sci (Lond) 93:127-135

18. Schneider SH, Khachadurian AK, Amorosa LF, Clemow L, Ruderman NB (1992) Ten-year experience with an exercise-based outpatient life-style modification program in the treatment of diabetes mellitus. Diabetes Care 15:1800-1810

19. Balducci S, Leonetti F, Di Mario U, Fallucca F (2004) Is a long-term aerobic plus resistance training program feasible for and effective on metabolic profiles in type 2 diabetic patients? Diabetes Care 27: 841-842

20. Hanefeld M, Fischer S, Schmechel H et al (1991) Diabetes Intervention Study. Multi-intervention trial in newly diagnosed NIDDM. Diabetes Care 14:308-317

21. Tudor-Locke C, Bell RC, Myers AM et al (2004) Controlled outcome evaluation of the First Step Program: a daily physical activity intervention for individuals with type II diabetes. Int J Obes Relat Metab Disord 28:113-119

22. Fritz T, Rosenqvist U (2001) Walking for exercise - immediate effect on blood glucose levels in type 2 diabetes. Scand J Prim Health Care 19:31-33

23. Sigal RJ, Kenny GP, Boule NG et al (2007) Effects of aerobic training, resistance training, or both on glycemic control in type 2 diabetes: a randomised trial. Ann Intern Med 147:357369

24. Bouma M, Rutten GE, de Grauw WJ, Wiersma T, Goudswaard AN (2006) [Summary of the practice guideline 'Diabetes mellitus type 2' (second revision) from the Dutch College of General Practitioners]. Ned Tijdschr Geneeskd 150:2251-2256

25. World Health Organization Expert Committee (1999) Definition, diagnosis and classification of diabetes mellitus and its complications. Part 1: Diagnosis and classification of diabetes mellitus. World Health Organization, Geneva

26. Zhang YY, Johnson MC 2nd, Chow N, Wasserman K (1991) Effect of exercise testing protocol on parameters of aerobic function. Med Sci Sports Exerc 23:625-630

27. Sigal RJ, Kenny GP, Wasserman DH, Castaneda-Sceppa C, White RD (2006) Physical activity/exercise and type 2 diabetes: a consensus statement from the American Diabetes Association. Diabetes Care 29:1433-1438

28. Byrne NM, Hills AP, Hunter GR, Weinsier RL, Schutz Y (2005) Metabolic equivalent: one size does not fit all. J Appl Physiol 99:1112-1119

29. Phillips WT, Ziuraitis JR (2004) Energy cost of single-set resistance training in older adults. J Strength Cond Res 18:606-609 
30. Storer TW, Davis JA, Caiozzo VJ (1990) Accurate prediction of $\dot{V} \mathrm{O}_{2 \max }$ in cycle ergometry. Med Sci Sports Exerc 22:704-712

31. Fairbarn MS, Blackie SP, McElvaney NG, Wiggs BR, Pare PD, Pardy RL (1994) Prediction of heart rate and oxygen uptake during incremental and maximal exercise in healthy adults. Chest 105:1365-1369

32. Matthews DR, Hosker JP, Rudenski AS, Naylor BA, Treacher DF, Turner RC (1985) Homeostasis model assessment: insulin resistance and beta-cell function from fasting plasma glucose and insulin concentrations in man. Diabetologia 28:412-419

33. Katsuki A, Sumida Y, Gabazza EC et al (2001) Homeostasis model assessment is a reliable indicator of insulin resistance during follow-up of patients with type 2 diabetes. Diabetes Care 24:362-365

34. Hays RD, Sherbourne CD, Mazel RM (1993) The RAND 36-item health survey 1.0. Health Econ 2:217-227

35. VanderZee KI, Sanderman R, Heyink JW, de Haes H (1996) Psychometric qualities of the RAND 36-Item Health Survey 1.0: a multidimensional measure of general health status. Int J Behav Med 3:104-122

36. Unnebrink K, Windeler J (2001) Intention-to-treat: methods for dealing with missing values in clinical trials of progressively deteriorating diseases. Stat Med 20:3931-3946

37. Kirk A, Mutrie N, MacIntyre P, Fisher M (2004) Effects of a 12month physical activity counselling intervention on glycaemic control and on the status of cardiovascular risk factors in people with Type 2 diabetes. Diabetologia 47:821-832

38. Haus JM, Carrithers JA, Trappe SW, Trappe TA (2007) Collagen, cross-linking, and advanced glycation end products in aging human skeletal muscle. J Appl Physiol 103:2068-2076
39. Kjaer M (2004) Role of extracellular matrix in adaptation of tendon and skeletal muscle to mechanical loading. Physiol Rev 84:649-698

40. Must A, Spadano J, Coakley EH, Field AE, Colditz G, Dietz WH (1999) The disease burden associated with overweight and obesity. Jama 282:1523-1529

41. Sturmer T, Brenner H, Brenner RE, Gunther KP (2001) Non-insulin dependent diabetes mellitus (NIDDM) and patterns of osteoarthritis. The Ulm osteoarthritis study. Scand J Rheumatol 30:169-171

42. Andersen H, Nielsen S, Mogensen CE, Jakobsen J (2004) Muscle strength in type 2 diabetes. Diabetes 53:1543-1548

43. Willey KA, Singh MA (2003) Battling insulin resistance in elderly obese people with type 2 diabetes: bring on the heavy weights. Diabetes Care 26:1580-1588

44. De Feyter HM, Praet SF, van den Broek NM et al (2007) Exercise training improves glycemic control in long-standing, insulintreated type 2 diabetes patients. Diabetes Care 30:2511-2513

45. West DS, DiLillo V, Bursac Z, Gore SA, Greene PG (2007) Motivational interviewing improves weight loss in women with type 2 diabetes. Diabetes Care 30:1081-1087

46. Kirk A, De Feo P (2007) Strategies to enhance compliance to physical activity for patients with insulin resistance. Appl Physiol Nutr Metab 32:549-556

47. Ivy JL (1997) Role of exercise training in the prevention and treatment of insulin resistance and non-insulin-dependent diabetes mellitus. Sports Med 24:321-336

48. Brandon LJ, Gaasch DA, Boyette LW, Lloyd AM (2003) Effects of long-term resistive training on mobility and strength in older adults with diabetes. J Gerontol A Biol Sci Med Sci 58:740-745

49. Pedersen BK, Saltin B (2006) Evidence for prescribing exercise as therapy in chronic disease. Scand J Med Sci Sports 16(Suppl 1):3-63 